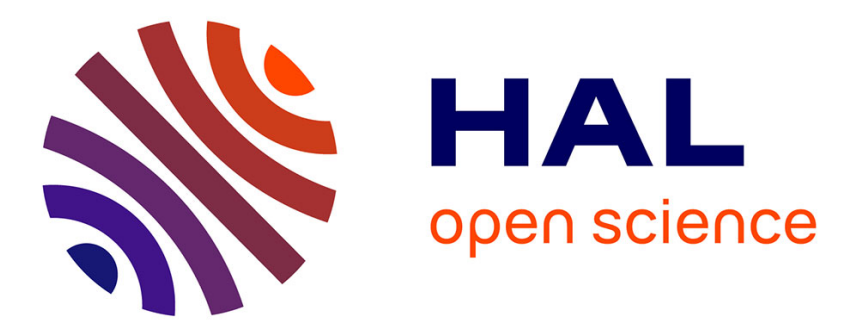

\title{
Spectral restoration in high resolution electron energy loss spectroscopy based on iterative semi-blind Lucy-Richardson algorithm applied to rutile surfaces
}

Rémi Lazzari, Jingfeng Li, Jacques Jupille

\section{- To cite this version:}

Rémi Lazzari, Jingfeng Li, Jacques Jupille. Spectral restoration in high resolution electron energy loss spectroscopy based on iterative semi-blind Lucy-Richardson algorithm applied to rutile surfaces. Review of Scientific Instruments, 2015, 86 (1), pp.013906. 10.1063/1.4905433 . hal-01442820

\author{
HAL Id: hal-01442820 \\ https://hal.science/hal-01442820
}

Submitted on 3 Jul 2020

HAL is a multi-disciplinary open access archive for the deposit and dissemination of scientific research documents, whether they are published or not. The documents may come from teaching and research institutions in France or abroad, or from public or private research centers.
L'archive ouverte pluridisciplinaire HAL, est destinée au dépôt et à la diffusion de documents scientifiques de niveau recherche, publiés ou non, émanant des établissements d'enseignement et de recherche français ou étrangers, des laboratoires publics ou privés. 


\section{Spectral restoration in high resolution}

electron energy loss spectroscopy based on iterative semi-blind Lucy-Richardson algorithm applied to rutile surfaces

Cite as: Rev. Sci. Instrum. 86, 013906 (2015); https://doi.org/10.1063/1.4905433

Submitted: 03 November 2014 . Accepted: 22 December 2014 . Published Online: 15 January 2015

Rémi Lazzari, Jingfeng Li, and Jacques Jupille
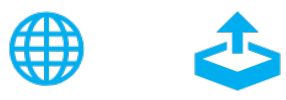

\section{ARTICLES YOU MAY BE INTERESTED IN}

Electron energy loss spectroscopy with parallel readout of energy and momentum Review of Scientific Instruments 88, 033903 (2017); https://doi.org/10.1063/1.4977529

\begin{tabular}{|c|c|c|c|c|}
\hline $\begin{array}{c}\text { Nanopositioning } \\
\text { Systems }\end{array}$ & $\begin{array}{c}\text { Modular } \\
\text { Motion Control }\end{array}$ & $\begin{array}{c}\text { AFM and NSOM } \\
\text { Instruments }\end{array}$ \\
\hline MAD CITY LABS INC. \\
Www.madcitylabs.com
\end{tabular}




\title{
Spectral restoration in high resolution electron energy loss spectroscopy based on iterative semi-blind Lucy-Richardson algorithm applied to rutile surfaces
}

\author{
Rémi Lazzari, 1,2,a) Jingfeng Li, ${ }^{1,2, b)}$ and Jacques Jupille ${ }^{1,2, c)}$ \\ ${ }^{1}$ CNRS, UMR 7588, Institut des NanoSciences de Paris, 75005 Paris, France \\ ${ }^{2}$ Sorbonne Universités, UPMC Univ Paris 06, UMR 7588, Institut des NanoSciences de Paris, 75005 Paris, \\ France
}

(Received 3 November 2014; accepted 22 December 2014; published online 15 January 2015)

\begin{abstract}
A new spectral restoration algorithm of reflection electron energy loss spectra is proposed. It is based on the maximum likelihood principle as implemented in the iterative Lucy-Richardson approach. Resolution is enhanced and point spread function recovered in a semi-blind way by forcing cyclically the zero loss to converge towards a Dirac peak. Synthetic phonon spectra of $\mathrm{TiO}_{2}$ are used as a test bed to discuss resolution enhancement, convergence benefit, stability towards noise, and apparatus function recovery. Attention is focused on the interplay between spectral restoration and quasi-elastic broadening due to free carriers. A resolution enhancement by a factor up to 6 on the elastic peak width can be obtained on experimental spectra of $\mathrm{TiO}_{2}(110)$ and helps revealing mixed phonon/plasmon excitations. (C) 2015 AIP Publishing LLC. [http://dx.doi.org/10.1063/1.4905433]
\end{abstract}

\section{INTRODUCTION}

High-resolution electron energy loss spectroscopy $(\text { HREELS })^{1}$ is a major surface science tool to probe vibrations of adsorbates and quantized low energy solid state excitations. HREELS is sensitive to small coverages, can probe excitation down to a few milli-electron volts and possesses a wide dynamical range and an angular resolution allowing dispersion measurements. Relying on selection rules of dipolar, impact, and resonant scattering, ${ }^{1,2}$ the analysis of vibrational frequencies and corresponding loss intensities enables the determination of adsorption sites, orientation of molecular axis, molecular or dissociative adsorption, and dispersion with parallel wavevector transfer. The main disadvantage of HREELS is its moderate resolution compared to that of other vibrational tools such as infra-red reflection absorption ${ }^{3}$ or non-linear spectroscopies. Some electron spectroscopy techniques such as ultraviolet photoemission are restricted by intrinsic bandwidths relative to shifts. In the same way as Xray photoemission, HREELS is among those that are mainly limited by instrumental factors, i.e., electron optics involving source, monochromator, analyzer, and focusing optics. ${ }^{4,5}$ Those limitations have prompted continuous improvements of electron optics design. ${ }^{4,6}$ Latest generations of instruments have reached a resolution lower than $1 \mathrm{meV}$ as measured by the full-width at half maximum (FWHM) of the elastic peak. ${ }^{7}$ However, it remains difficult to go routinely below 4-5 meV not only because of electron optics and loss in intensity due to monochromatization but also because of the physics of scattering including sample reflectivity and continuous loss processes.

\footnotetext{
a) Author to whom correspondence should be addressed. Electronic mail: remi.lazzari@insp.jussieu.fr

b)Electronic mail: jingfeng.li@insp.jussieu.fr

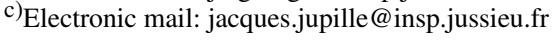

To improve interpretations that are often hindered by overlapping features, numerical deconvolution of intense multiple/combination Fuchs-Kliewer phonons, ${ }^{8}$ in particular, in oxides, has proven its interest in deciphering hidden molecular vibrations $^{9,10}$ and removing multiple losses. ${ }^{11}$ But spectral restoration methods to improve the apparent resolution have been poorly explored. The suitable template of the transfer function or point spread function (PSF) provided by the elastic peak favors the application of these methods to HREELS. McKenna and Apai ${ }^{12}$ used commercially available maximum likelihood routines to compare infra-red and EELS vibrational frequencies of polymer layers. Similar resolution enhancement algorithm was applied by Frederick et al. to assign adsorbate vibrations on copper ${ }^{13}$ and to support dielectric interpretation of phonon modes in supported thin alumina films. ${ }^{14}$ Wang and Weinberg ${ }^{15,16}$ implemented Burg maximum entropy method ${ }^{17}$ to the case of $\mathrm{CO}$ adsorbed on $\mathrm{Ru}(001)$. The deconvolution performed in Fourier domain is based on a spectral estimation using maximum entropy method that assumes Lorentzian line-shape. Astonishingly, this methodology of ultra-high resolution was never thereafter used. More conventional maximum entropy analysis was used to derive surface phonon modes of $\mathrm{p}(2 \times 2) \mathrm{Ni}(111)$ buried in the elastic peak, ${ }^{18}$ identify $\mathrm{CO}$ adsorption sites and alloy composition on $\mathrm{Pt}_{2} \mathrm{Ni}_{1-x}(111),{ }^{19}$ unravel reaction intermediates in thermal decomposition processes of molecules, ${ }^{20}$ or study surface hydrogenation. ${ }^{21,22}$ Kesmodel et $a .^{23}$ showed that the modest resolution on bulk polymer samples can be safely improved by a factor 2 using Lucy-Richardson (LR) maximum likelihood approach. Spectral restoration was also fruitful in the case of overlapping vibrational modes of thin film of molecules: $\mathrm{C}_{60}$ on $\mathrm{InP}(001),{ }^{24}$ amino-acids on $\mathrm{Au}(111) .{ }^{25}$ In a series of papers about potentialities of wavelets analysis, Charles et $a l{ }^{26}$ tackled among other the case of HREELS but without any application to actual data. Frederick et al. reviewed the deconvolution strategies and pitfalls in the context of HREELS, ${ }^{5}$ 
and proposed a method to remove continuous and multiple losses from the substrate to enhance the resolution of discrete lines. ${ }^{11}$

The widespread problem of PSF deconvolution in physics has led to the refinement of numerous numerical methods, ${ }^{17,27,28}$ in particular, in the context of image analysis. The first category of algorithms involves Fourier transform and deconvolution by division in reciprocal space with suitable filtering/regularization (such as Wiener algorithm) or maximum entropy procedures to stabilize the inversion against noise amplification. The second class relies on maximum likelihood principle to optimize the deconvolution by using extra information on the noise statistics. This uses only multiplications in Fourier space and iterative algorithms among which the wellknown LR algorithm..$^{29,30}$ Originally devised to suppress the deleterious effects of image blurring due to telescopes aberrations in astronomy, it was intensively used in this context. The approach seeks the maximum likelihood between the unbroadened object and the measurement subject to Poisson counting statistics. It was then successfully applied to several domains of physics such as optical microscopy, ${ }^{27,31}$ EELS in transmission electron microscopy, ${ }^{32,33}$ electron diffraction, ${ }^{34} \mathrm{X}$-ray absorption spectroscopy, ${ }^{35}$ photoemission spectroscopy, ${ }^{36}$ and grazing incidence small-angle $x$-ray scattering. ${ }^{37}$

The article is divided as follows: after introducing experimental conditions, the semi-blind LR algorithm, its interest and its numerical implementation are presented. The robustness of the analysis is then tested on synthetic phonon spectrum of titania. Resolution enhancement (a factor 6 can be achieved starting with an elastic FWHM of $12 \mathrm{meV}$ ), convergence benefit, stability towards noise level, PSF shape recovery, and interplay between PSF and low energy excitations are in turn theoretically discussed. Finally, the method is applied to experimental spectra of multiple excitations in reduced $\mathrm{TiO}_{2}$ to determine whether the donors at the origin of its $n$ type conductivity can be observed by electron energy loss spectroscopy.

\section{EXPERIMENTAL}

Experiments have been performed in a vacuum vessel (base pressure in the high $10^{-9} \mathrm{~Pa}$ ) equipped with a combined 4-grids LEED (Low Energy Electron Diffraction)/Auger device and a HREELS spectrometer (LK-2000 from LKTechnology). The phonon spectrum of $\mathrm{TiO}_{2}(110)$ has been used as a test case for the LR resolution enhancement. HREELS spectra were acquired at room temperature on a spectrometer possessing a dual $127^{\circ}$ cylindrical monochromator and a rotatable analyzer. ${ }^{38} \mathrm{~A}$ total scattering angle of $120^{\circ}$ in the specular geometry (incident angle $\theta_{I}=60^{\circ}$ ) was used for all spectra. The beam of energy $E_{i}=8.8 \mathrm{eV}$ was scattered approximately either parallel or perpendicular to the bridging oxygen [001] direction of $\mathrm{TiO}_{2}(110)$. A counting rate of a few $10^{5}$ was routinely achieved with elastic FWHM from 9 to $20 \mathrm{meV}$. Several cycles of sputtering-annealing $\left(850 \mathrm{eV} \mathrm{Ar}^{+}\right.$ions/ $\left.T \simeq 1100 \mathrm{~K}\right)$ of rutile $\mathrm{TiO}_{2}(110)$ surfaces were required to achieve a sharp LEED $(1 \times 1)$ pattern and a sample free of contaminants, as judged by Auger spectroscopy and HREELS through the absence of
$\mathrm{CH}$-stretching frequencies and elastic peak intensity. Titania substrates were conductive enough as visually checked by their blue color to avoid any charge compensation during the EELS measurements.

\section{RESOLUTION ENHANCEMENT VIA SEMI-BLIND LUCY-RICHARDSON ALGORITHM}

\section{A. Theoretical background}

The measured HREELS spectrum $I(x)$, where $x=\hbar \omega$ is the energy loss, may be approximated as a convolution of the object spectrum $O(x)$ (the multiple losses/gains spectrum) with the total PSF $P(x)$ of the apparatus optics including the defects of the sample, ${ }^{5}$ plus an additive counting noise of Poisson distribution $N(x)$ to account for the random arrival of electrons on the detector ${ }^{4}$

$$
I(x)=P(x) \otimes O(x)+N(x) .
$$

The convolution product is defined as $(P \otimes O)(x)=\int P(x$ $-y) . O(y) d y . P(x)$ is also known as the transfer or resolution function. It fulfills the normalization condition $\int P(x) d x$ $=1$. The alteration of the resolution with increasing energy loss due to aberrations along beam path will be disregarded in the following. Fourier transform of Eq. (1) reads $I(u)$ $=P(u) . O(u)+N(u)$, where $u$ is the reciprocal variable of $x$ and $P(u), O(u), N(u)$ the corresponding transforms. The presence of noise precludes the naive direct deconvolution of the PSF through back Fourier transform of $O(u)=\frac{I(u)}{P(u)}-\frac{N(u)}{P(u)}$. Indeed, the noise contribution is by essence not known exactly and the decay (if any !) of its Fourier components $|N(u)|$ with $u$ is much lower than that of the signal leading to their amplification in the back Fourier process if noise is neglected. Strategies have been designed all along the years to partially circumvent this problem of Fourier deconvolution such as Wiener filtering approach. ${ }^{39}$ But iterative algorithms turned out to have decisive advantages since they involve only multiplication in the Fourier domain.

The LR algorithm ${ }^{29,30}$ has become popular in the field of astronomy and medical imaging. It uses an extra piece of information regarding the nature of the noise distribution. For a Poisson statistics, the probability of having a given signal $I$ knowing the object function $O$ reads

$$
P[I \mid O]=\prod_{x} \frac{[P(x) \otimes O(x)]^{I(x)} \exp [-P(x) \otimes O(x)]}{I(x) !} .
$$

The Bayses theorem of statistics $p[O \mid I]=P[I \mid O] P[O] / P[I]$ gives access to the quantity of interest $p[O \mid I]$, the probability of having the object $O$ knowing the signal $I$. The maximum likelihood solution maximizes only the density $p[I \mid O]$ at constant $P(I)$ and for a uniform probability density $p(O)$. By maximizing the logarithm of Eq. (2) as function of the object, a closed equation is obtained

$$
\left[\frac{I(x)}{P(x) \otimes O(x)}\right] \otimes P(-x)=\delta(x) .
$$

It can be mapped onto an iterative self-consistent process which links the object function to its value at a previous 
iteration $i$

$$
O_{i+1}(x)=\left\{\left[\frac{I(x)}{P(x) \otimes O_{i}(x)}\right] \otimes P(-x)\right\} O_{i}(x),
$$

with $i=1, \ldots, N_{O}$ and the often used initial condition $O_{0}(x)$ $=I(x)$. The iterative LR algorithm has numerous advantages. The total intensity is conserved at each iteration $\int O_{i}(x) d x$ $=\int I(x) d x$, meaning that intensity is neither created nor destroyed. The procedure which is free of adjustable parameters guarantees also that $O_{i}(x) \geq 0, \forall i$, a compulsory condition for a physical signal which is not always fulfilled by other deconvolution techniques. The algorithm is relatively stable regarding noise and small errors in the PSF although at some point it will amplify them. A strength of the iterative approach over Fourier based methods is the possibility of applying, at each step, an operator $\mathcal{F}$ such as symmetry constraints, ${ }^{34}$ prior information, filtering, regularization, and normalization

$$
O_{i+1}(x)=\mathcal{F}\left\{x, O_{i}(x),\left[\frac{I(x)}{P(x) \otimes O_{i}(x)}\right] \otimes P(-x)\right\} .
$$

In particular, special regularization schemes ${ }^{31,37}$ have been developed to remove noise while preserving signal. In LR deconvolution, there is no predefined convergence criterium which is always a compromise between resolution enhancement and noise amplification. To weigh up the pro and cons of the approach, attention must be paid to drawbacks such as ripples close to intense features, ${ }^{33}$ local gradient convergence rate, ${ }^{40}$ boundary effects on size limited data, ${ }^{41}$ importance of the sampling step, and noise amplification. ${ }^{33}$

Up to now, the PSF $P(x)$ was implicitly assumed to be known. Good approximation of the PSF in HREELS, ${ }^{4}$ the direct through peak cannot always be measured. Low energy tails of the elastic peak due to plasmon, ${ }^{42}$ combination bands or electron-hole pair excitations ${ }^{43}$ and sample roughness prevent the direct use of the elastic peak as a template for PSF. ${ }^{5}$ But, if one realizes that the PSF $P(x)$ and the object $O(x)$ play a symmetric role regarding the intensity and noise in Eq. (1), a so-called blind deconvolution and restoration ${ }^{41,44}$ of both $P(x)$ and $O(x)$ can be built. At given steps, the deconvoluted object $O^{k-1}(x)$ is used as the input of the iterative LR loop for the PSF and, in a symmetrical way, the deconvoluted PSF $P^{k-1}(x)$ is used as the input of the iterative LR loop for the object

$$
\begin{aligned}
& O_{i+1}^{k}(x)=\left\{\left[\frac{I(x)}{P^{k-1}(x) \otimes O_{i}^{k}(x)}\right] \otimes P^{k-1}(-x)\right\} O_{i}^{k}(x) \\
& \text { with } i=1, \ldots, N_{O} \\
& \kappa P_{j+1}^{k}(x)=\left\{\left[\frac{I(x)}{O^{k-1}(x) \otimes P_{j}^{k}(x)}\right] \otimes O^{k-1}(-x)\right\} P_{j}^{k}(x) \\
& \text { with } j=1, \ldots, N_{P} \\
& \int P_{j+1}^{k}(x) d x=1 .
\end{aligned}
$$

$i=1, \ldots, N_{O}$ and $j=1, \ldots, N_{P}$ are the indexes of the standard LR loop for object and PSF, respectively; $k=1, \ldots, N_{B}$ is the index of the upper blind deconvolution. At each step, a normalization factor $\kappa$ is applied to $P_{j+1}^{k}(x)$ to comply with the normalization of the PSF (Eq. (8)) and to avoid intensity transfer from the object.

Because of the symmetrical roles of $P(x)$ and $O(x)$, there is no unique solution to the problem and the iterative procedure is still sensitive to the starting point (see below). However, by starting from a fair guess of the PSF (for instance, the elastic peak $\left.P_{0}^{0}=\mathcal{P}\{I(x)\}\right)$ or by imposing some constraints on the PSF (such as the peak shape or the support of the function ${ }^{44}$ ) or on the final solution (such as the Boltzmann ratio of gain and loss peaks ${ }^{5}$ ), one expects to recover the most probable shape of the PSF in the maximum likelihood wise. Also, it turns out that the convergence speed slows down dramatically with indexes $i$ and $j$, as it is well known for LR deconvolution, as well as with the index $k$ because the algorithm transfers intensity from object to PSF. This slow down can be circumvented by resetting periodically in Eqs. (6)-(8), the intensity $I(x)$ and the PSF by the deconvoluted object $O^{k}(x)$ and a peak whose width is close to that of the "elastic peak" of $O^{k}$, respectively. Therefore, the used a priori knowledge is that the zero loss $x=0$ intensity tends towards a Dirac peak. Formally, this extra-loop reads

$$
\begin{aligned}
I[l+1]_{0}^{0}(x)= & O[l]_{N_{O}}^{N_{B}}, \quad P[l+1]_{0}^{0}(x)=\mathcal{P}\left\{O[l]_{N_{O}}^{N_{B}}\right\} \\
& \text { with } l=0, \ldots, N_{I} .
\end{aligned}
$$

This repeated deconvolution is the heart of the proposed algorithm. Introducing this a priori knowledge $(\mathcal{P}$ operator in Eq. (9)) regarding the shape and the nature of the PSF forces, the convergence towards a PSF solution whose width is close to the initial elastic peak while having its shape determined by the algorithm. This allows the extraction of loss features which are close to the elastic peak and a considerable speeding up of the deconvolution ${ }^{41}$ (see below). The final total point spread function is defined through the folding product of all the intermediate PSFs: $P_{t}(x)=\bigotimes_{l=1}^{N_{I}} P[l]_{N_{P}}^{N_{B}}(x)$.

\section{B. Numerical implementation}

Equations (6) and (7) are solved iteratively with the initial condition $O[0]_{0}^{0}(x)=I(x)$. The starting PSF function $(\mathcal{P}$ operator in Eq. (9)) is taken as a peak (in general, a Gaussian) whose FWHM is equal to a fraction of the elastic peak $O[l]_{N_{O}}^{N_{B}}$ to be deconvoluted. Since $I, P, O$ quantities are defined on $N$ equally spaced abscissae $x_{n}$, the convolution products are discrete sums. Although some solutions have been proposed to control the flow of intensity at the boundaries in the LR algorithm, ${ }^{41}$ the adoption of zero padding of vectors led to negligible artefacts close to the limits $x_{0}, x_{N}$. As done herein, noise amplification can be damped out by folding the PSF at the end of the blind deconvolution process Eqs. (6) and (7), i.e., $O[l]_{N_{O}}^{N_{B}}$, with a Gaussian peak whose FWHM is a fraction of the PSF. The four integers which control the convergence $\left(N_{I}, N_{B}, N_{O}, N_{P}\right)$ will be used hereafter to label each deconvolution process. Notice that $\left(1, N_{B}, 1,0\right)$ stands for standard LR algorithm for which the initial PSF is assumed to be known and fixed. For symmetry reasons, $N_{O}=N_{P}$ was systematically used in our semi-blind approach. The figures of merit $\chi^{2}$ and $R$ characterize the agreement between the initial intensity, total deconvoluted PSF, and final 
spectrum

$$
\begin{aligned}
& \chi^{2}\left[l k\left(N_{O}+N_{P}\right)\right] \\
& \quad=\frac{1}{N} \sum_{n}\left\{\left[P[l]_{N_{P}}^{k} \otimes O[l]_{N_{O}}^{k}\right]\left(x_{n}\right)-I\left(x_{n}\right)\right\}^{2} / I\left(x_{n}\right), \\
& R\left[l k\left(N_{O}+N_{P}\right)\right] \\
& \quad=\sum_{n}\left|\left[P[l]_{N_{P}}^{k} \otimes O[l]_{N_{O}}^{k}\right]\left(x_{n}\right)-I\left(x_{n}\right)\right| / \sum_{n}\left|I\left(x_{n}\right)\right| .
\end{aligned}
$$

$\chi^{2}$ is statistically meaningful, despite its underlying normal statistics since LR algorithm is intrinsically a maximum likelihood method. Although precise convergence criteria regarding compromise between object restoration and noise amplification are difficult to define, FWHMs of the local $\left(P[l]_{N_{P}}^{k}\right)$ and total $\left(P_{t}[l](x)\right)$ PSFs and of the elastic peak of the object $O[l]_{N_{O}}^{k}$ are used herein to characterize the signal enhancement.

\section{NUMERICAL TESTS OF THE ALGORITHM ON SYNTHETIC HREELS SPECTRA}

Before any application to experimental data, synthetic HREELS spectra of titania for which the sought solution is perfectly known are used to test the algorithm, in terms of convergence benefit, stability towards noise level, and PSF shape recovery.

\section{A. Calculation of HREELS synthetic data}

Simulated HREELS spectrum of the Fuchs-Kliewer phonons and semiconductor Drude-like losses ${ }^{45,46}$ in rutile $\mathrm{TiO}_{2}$ have been used as synthetic data to test the above LR deconvolution technique. In the dipole scattering condition which dominates in the specular geometry, ${ }^{1}$ the cross-section of single excitation loss $S(\hbar \omega)$ for bulk material ${ }^{1,2,47,48}$ is related to its dielectric function $\epsilon_{B}(\omega)$ through

$$
\begin{aligned}
S(\hbar \omega)= & \frac{4\left|R_{I}\right|^{2}\left(1-2 \theta_{E}\right)^{1 / 2}}{\pi^{2} a_{0} k_{I} \cos \theta_{I}} \frac{1+n(\omega)}{\hbar \omega} \\
& \times \operatorname{Im}\left[\frac{-1}{1+\epsilon_{b}(\omega)}\right] F_{b}(\hbar \omega) .
\end{aligned}
$$

$E_{I}=\hbar^{2} k_{I}^{2} / 2 m$ is the impact energy of the electron of wavevector $k_{I}$ and mass $m$ which impinges the sample surface at angle of incidence $\theta_{I} . \theta_{E}=\hbar \omega / 2 E_{I}$ is a reduced variable which defines the angular aperture of dipole scattering. $a_{0}$ is the Bohr radius while $R_{I}$ is the reflection coefficient of the electrons at the sample surface, hereafter assumed to be one. $S(\hbar \omega)$ is also modulated by the Bose statistics $n(\omega)$, i.e., the population of excitations at a given temperature ( $T=300 \mathrm{~K}$ herein). The cross section is multiplied by the sensitivity function of the apparatus $F_{b}(\hbar \omega)$ which is due to the wavevector integration over the detection slit. Although the focusing optics of our apparatus prevents its easy calculation, it was estimated for a circular aperture ${ }^{1}$ defining an acceptance angle $\theta_{C}$

$$
F_{b}\left(\hbar \omega, \theta_{C}\right)=\frac{1}{\theta_{E}^{2}} \int_{0}^{\hat{\theta}_{C}} d \hat{\theta} \int_{0}^{2 \pi} d \phi
$$

$$
\times \frac{\left[\left(\sin \theta_{I}-\hat{\theta} \cos \theta_{I} \cos \phi\right)^{2}+\hat{\theta}^{2} \sin ^{2} \phi\right]^{1 / 2}}{\left[1+\hat{\theta}^{2}\right]^{2}},
$$

where

$$
\hat{\theta}=\theta / \theta_{E}, \hat{\theta}_{C}=\theta_{C} / \theta_{E} .
$$

A typical value of $\theta_{C}=1.5^{\circ}$ was used for the simulated spectrum to account for the damping of the peak intensity with energy loss (inset of Fig. 1).

Only four modes are dipole active among the 15 normal modes in bulk quadratic rutile, 3 of symmetry $E_{u}$, and 1 of symmetry $\mathrm{A}_{2 u} \cdot{ }^{49}$ The complex bulk dielectric function is taken as the sum of damped oscillators corresponding to infra-red or dipole active transverse phonons and eventually of a plasmonlike excitation at low energy which mimics the electronic contribution of defects, mainly oxygen vacancies and Ti interstitial $^{50}$

$$
\epsilon_{b}(\omega)=\epsilon_{\infty}-\frac{\omega_{P}^{2}}{\omega^{2}-i \omega \gamma_{P}}+\sum_{n} \frac{\omega_{p, n}^{2}}{\omega_{n}^{2}-\omega^{2}-i \omega \gamma_{n}} .
$$

$\omega_{n}$ is the transverse frequency of phonon $n, \gamma_{n}$ its damping and $\omega_{p, n}^{2}$ its oscillator strength while $\omega_{P}$ and $\gamma_{P}$ stand for the plasmon frequency and its damping. Residual contribution of all other types of excitations is included into the constant value ${ }^{49,51} \epsilon_{\infty}=6.5$. Fortunately, the optical anisotropy of the rutile structure can be neglected in HREELS due to a nearly degeneracy between the highest energy $\mathrm{E}_{u}$ mode and the $\mathrm{A}_{2 u}$ mode. ${ }^{49}$ This avoids to account for the orientation of the crystal axes relative to the scattering plane ${ }^{49,52}$ and allows the description of $S(\hbar \omega)$ as a simple sum over three oscillators. The numerical values $\omega_{p, n}=204.9 ; 50 ; 87.7 \mathrm{meV}$, $\gamma_{n}=22.7 ; 48.1 ; 62 \mathrm{meV}$, and $\gamma_{n}=2.27 ; 1.44 ; 1.55 \mathrm{meV}^{49}$ are extracted from infra-red data ${ }^{53}$ and confirmed by inelastic neutron scattering ${ }^{54}$ and density functional theory. ${ }^{55}$ Multiple excitations and combination modes following a Poisson statistics $^{1,48,56}$ are introduced through a self-convolution product of the single excitation

$$
\begin{aligned}
S_{m}(\hbar \omega)= & \delta(\hbar \omega)+S(\hbar \omega)+\frac{1}{2 !} S(\hbar \omega) \otimes S(\hbar \omega) \\
& +\frac{1}{3 !} S(\hbar \omega) \otimes S(\hbar \omega) \otimes S(\hbar \omega)+\cdots,
\end{aligned}
$$

$\delta(\hbar \omega)$ is the elastic peak. The final synthetic data $I(\hbar \omega)$ $=P(\hbar \omega) \otimes S_{m}(\hbar \omega)$ are obtained after convolution by an apparent PSF $P(\hbar \omega)$ which is assumed to be constant over all the frequency range. Of course, the two last operations are much more easily handled in reciprocal/temporal space.

The use of a Drude-like dielectric function to account for carriers deserves discussion in the light of polaronic interaction in $\mathrm{TiO}_{2}$. The $n$-type conductivity of reduced titania is not easily reconciled with the apparent deep character of the defect-induced band gap states that are found ca. $1 \mathrm{eV}$ below the Fermi level. Answers come by accounting for the vertical and adiabatic transitions associated to the defect levels. ${ }^{57,58}$ Vertical transitions, such as probed by photoemission and retain the polaronic geometry of the initial state, defects appear as deep donors although thermal activation energy for ionization can be much smaller. ${ }^{59}$ Adiabatic transition 


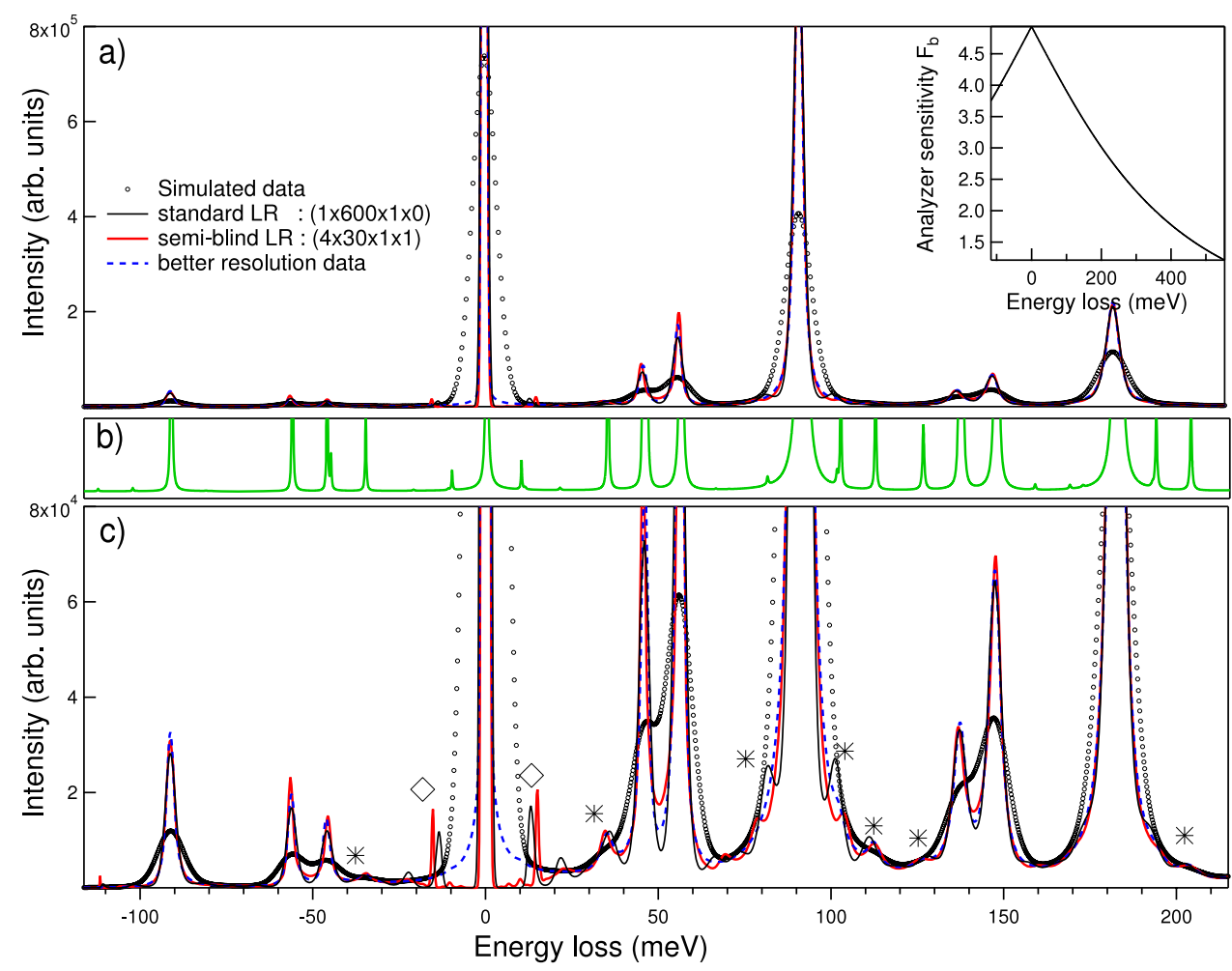

FIG. 1. (a)-(c) Calculated HREELS spectrum of titania with a FWHM $=8 \mathrm{meV}$ Gaussian PSF (circles) and LR deconvoluted spectra with the standard algorithm $(1 \times 600 \times 1 \times 0)$ (thin black line) and the semi-blind algorithm $(4 \times 30 \times 1 \times 1)$ (bold red line). Both are compared to a simulated spectrum (dotted blue line) with a better resolution FWHM $=1.6 \mathrm{meV}$ giving the same elastic peak width. The bottom panel (c) allows zooming on the peak feet of Fig. (a). Fig. (b) shows the expected line positions through an hypothetical simulation with arbitrary small phonon dampings $\gamma_{n}$. The upper right inset shows the analyzer sensitivity $F_{b}\left(\hbar \omega, \theta_{C}\right)$ (Eq. (12)) used in the simulation $\left(\theta_{C}=1.5^{\circ}\right)$. Star symbols highlight the features resolved by the algorithm while diamonds pinpoint deconvolution artifacts.

states involved in electron transport implies the relaxation of polarization and much shallower donors very close to conduction band. Thus, infrared reflectivity data of reduced rutile could be well fitted by introducing a plasmon contribution. ${ }^{45,60}$ Effective masses quite similar to those deduced from transport measurements were found. ${ }^{45,61,62}$ Above $250 \mathrm{~K}$, transport properties of $\mathrm{TiO}_{2}{ }^{63}$ are well accounted for by donor centers with an activation energy of $10 \mathrm{meV}$. The coexistence of polaron with free-carriers under photoexcitation or after doping, which was evidenced by infrared on powders ${ }^{64}$ was recently supported by theoretical approaches. ${ }^{58}$ In the $\mathrm{THz}$ range, experimental findings described by the Feynman polaron theory within the intermediate coupling regime were found compatible with a Drude behavior with a strong temperature dependent damping. ${ }^{46}$

To conclude, titania HREELS spectrum (Figs. 1 and 2) is an interesting test bed for the LR deconvolution because of the presence of two overlapping surface phonons at $\omega_{s, 1-2}$ $=46 ; 56.5 \mathrm{meV}$ of a close-by one at $\omega_{s, 3}=91 \mathrm{meV}$ and of combination and multiple excitations which span a large range of relative intensities and peak widths.

\section{B. Resolution enhancement and convergence benefit}

A deconvolution applied to a simulated spectrum obtained with a Gaussian PSF of FWHM=8 meV, without noise (see Sec. IV C), is shown in Figs. 1 and 2. Resolution, counting statistics $\left(7.4 \times 10^{5}\right.$ counts on the elastic peak), and energy step $(\Delta \hbar \omega=0.2 \mathrm{meV})$ are typical to experiments. Standard LR algorithm $(1 \times 600 \times 1 \times 0)$ is compared to semi-blind $(4 \times 30 \times 1 \times 1)$ with periodic resetting of the PSF. Notice that the initial elastic peak shape is exactly the sought solution. Whatever the approach, algorithms lead to a sizable resolution enhancement. The elastic peak FWHM is reduced from $8 \mathrm{meV}$ to $1.6 \mathrm{meV}$, a reasonable end value considering the step size $\Delta \hbar \omega$. A comparison with a spectrum calculated with a much better resolution (FWHM=1.6 meV) demonstrates that the deconvolution reproduces satisfactorily peak shapes and positions, in particular, for overlapping features generated by multiple excitations (Eq. (14)) (Figs. 1 and 2). While the broadness of the peaks at high energy obviates the interest of the resolution enhancement, the LR process is able to recover unapparent combination phonons (see stars in Figs. 1(b) and 2(b)) such that $\pm\left(\omega_{s, 3}-\omega_{s, 2}\right)=35 \mathrm{meV}$ or $\omega_{s, 3}+\omega_{s, 2}-\omega_{s, 1}$ $=102.5 \mathrm{meV}$. However, LR deconvolution gives rise to unphysical small ripples or wings ${ }^{33}$ (i) close to the most intense peaks, i.e., the elastic peak and the phonon at $\omega_{s, 3}=91 \mathrm{meV}$ (with, in addition, wrong positioning: $\pm\left(\omega_{s, 3}-\omega_{s, 2}\right)=10 \mathrm{meV}$ (diamond in Fig. 2(c))) or (ii) at the energy edges (diamond symbol in Fig. 1(b)). Techniques ${ }^{41}$ exist to overcome this last issue and to control the flow of intensity at the boundaries.

The first benefit of the semi-blind approach over nominal deconvolution shows up in the evolution of (i) the elastic peak FWHMs and (ii) of the figures of merit $R$ and $\chi^{2}$ (Fig. 3) with the numerical cost, i.e., the total of number of LR loops $N_{L R}$ $=N_{I} N_{B}\left(N_{O}+N_{P}\right)$. Much faster convergence is obtained with 


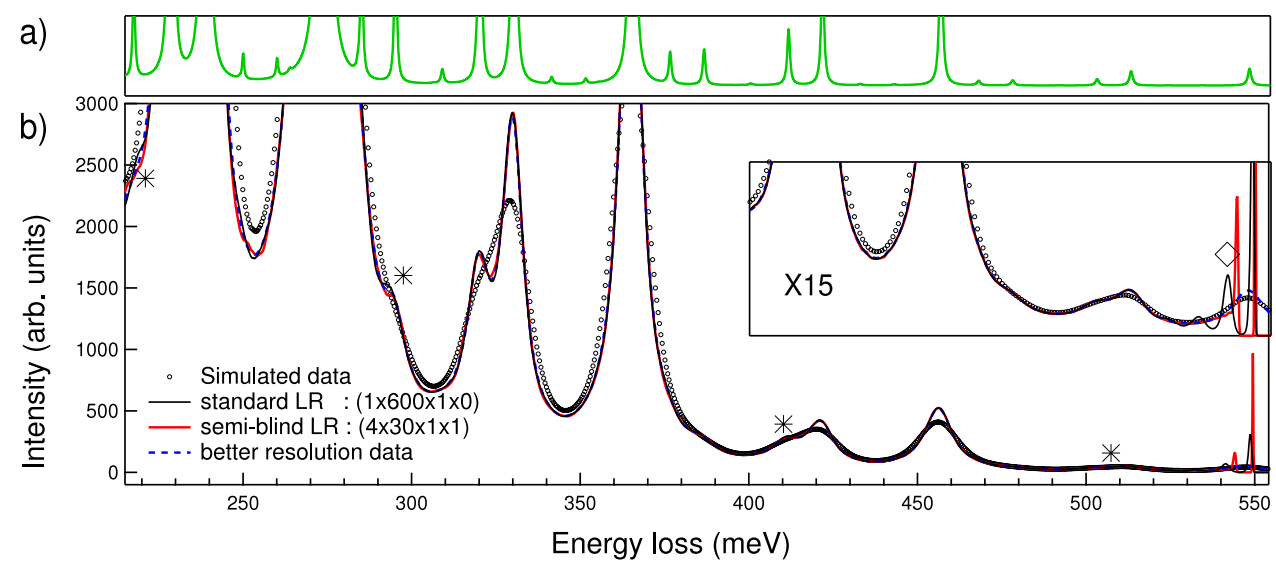

FIG. 2. Same as Fig. 1 but on the $220-550 \mathrm{meV}$ energy loss scale. The inset shows a zoom $(\times 15)$ of the spectra. Ripples appear after deconvolution at the edge of the spectrum due to finite size windowing.

the semi-blind methods (cases $(1 \times 120 \times 1 \times 1)$ and $(4 \times 30 \times 1$ $\times 1)$ ) than with the standard LR algorithm $(1 \times 600 \times 1 \times 0)$ which relies on the knowledge of the PSF. However, without resetting the PSF, the FWHM of the object elastic peak saturates $($ case $(1 \times 120 \times 1 \times 1))$ probably because the algorithm mixes object and PSF. Resetting the input of the LR cycles (Eq. (9)) cures the problem by reintroducing some knowledge of what the PSF should look like. This is at the expense of the saturation of the agreement factor, which remains however one order of magnitude lower than the standard LR method $(1 \times 600 \times 1 \times 0)$.

\section{Stability towards noise level}

Stability towards noise level is tested in a stringent way on simulated data with poor counting statistics (elastic peak maximum of $2 \times 10^{3}$ ) which may be typical of adsorbate vibration and involving a Poisson noise (Fig. 4(a), circle). The quality of restoration is a trade-off between signal and noise. Semi-blind deconvolutions applied to clean, noisy, and filtered data are compared in Fig. 4. As it is well known for the LR algorithm, the iterative process amplifies fluctuations of noisy data (Fig. 4(a), red line), even well above those of the initial data, with a poor benefit in term of resolution enhancement. This drawback is inherent to the maximum likelihood approach. Similar amplification would be obtained for high counting rates, in particular, in the high energy loss range because of the signal damping by the analyzer. To circumvent this issue, filtering or smoothing is required. Already a basic low band-pass filtering of the initial data improves noticeably the situation as shown by comparing the deconvolution of the clean data (Fig. 4(a), orange line) to the pre-filtered data

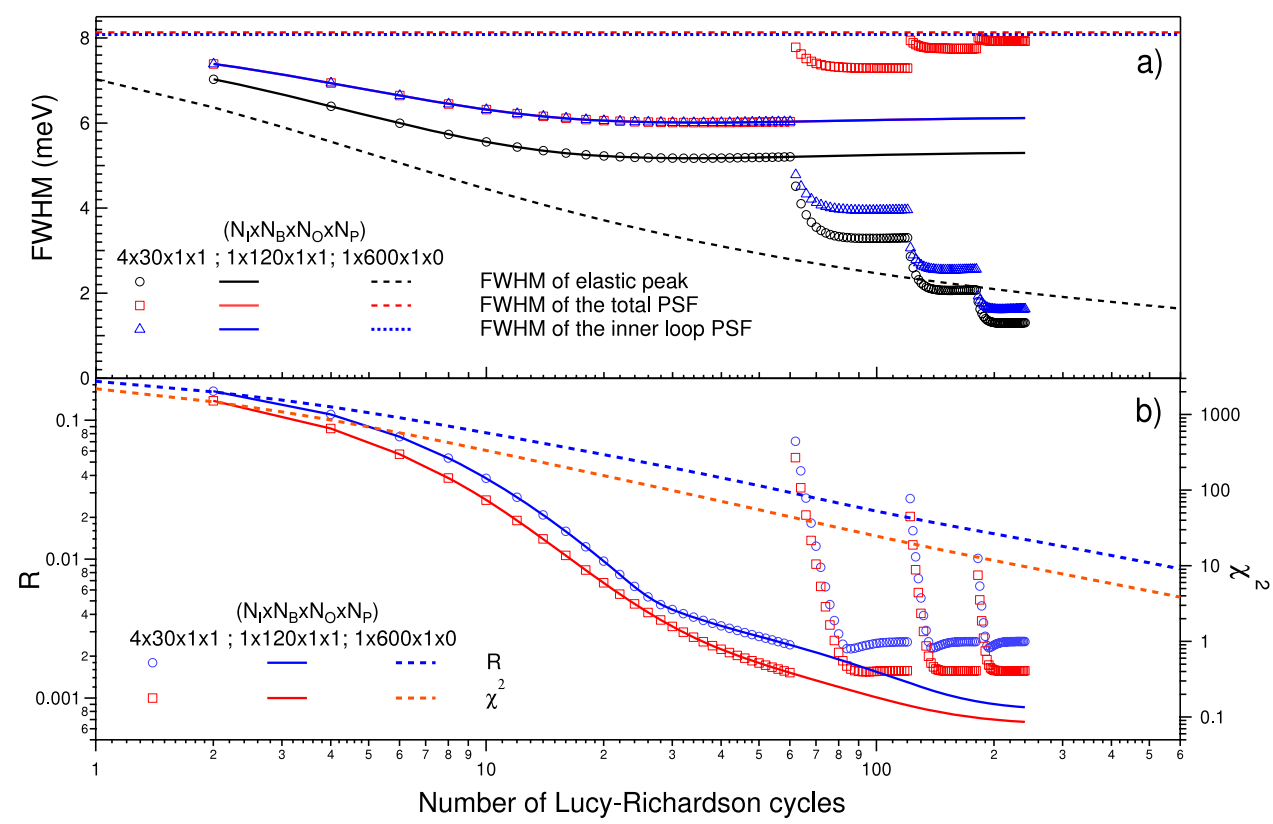

FIG. 3. Evolution as function the total number of LR cycles $N_{L R}=l k\left(N_{O}+N_{P}\right)$ of (a) the FWHMs of the elastic peak of the spectrum $O[l]_{N_{O}}^{k}$, of the total PSF $P_{t}$, and of the inner loop one $P[l]_{N_{P}}^{k}$ and of (b) the figures of merit (Eqs. (10)) of the deconvolution process $\left(R\right.$-left scale; $\chi^{2}$-right scale). Extended blind deconvolution $(1 \times 120 \times 1 \times 1)$ is compared to the one with periodic resetting of the PSF $(4 \times 30 \times 1 \times 1)$ and the standard LR algorithm $(1 \times 600 \times 1 \times 0)$. Agreement factors have been computed on the whole energy loss scale -115 to $550 \mathrm{meV}$. 

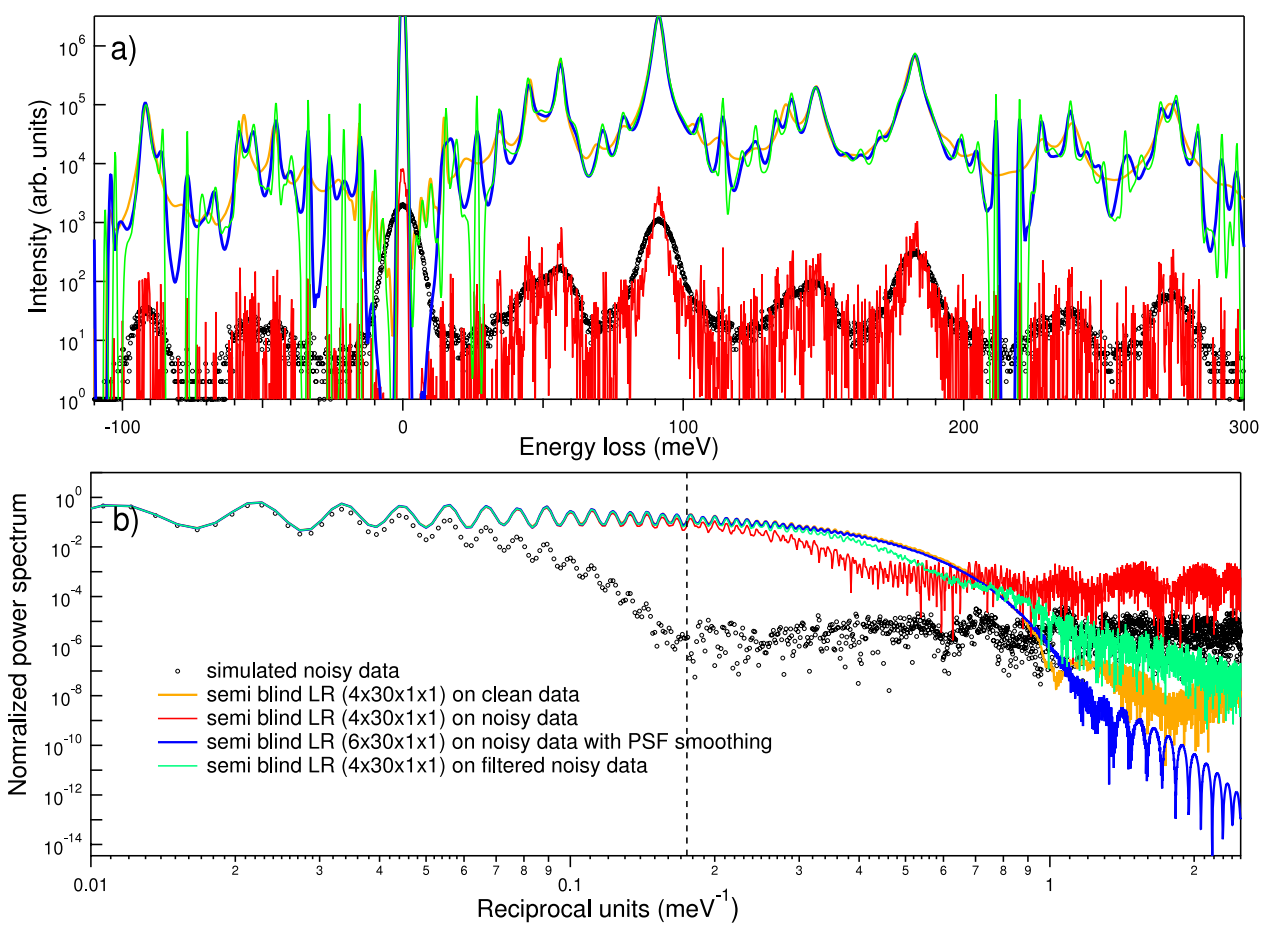

FIG. 4. (a) Simulated noisy HREELS spectrum of titania (circles; Gaussian PSF with FWHM $=8 \mathrm{meV}$ ) with a much poorer counting statistics $\left(2 \times 10^{3}\right.$ at elastic peak) than in Fig. $1\left(7.4 \times 10^{5}\right.$ at elastic peak). The noise follows a Poisson statistics. $(4 \times 30 \times 1 \times 1)$ semi-blind LR deconvolution is applied to clean (orange line) and noisy (red line) data. They are compared to $(6 \times 30 \times 1 \times 1)$ iterative processes including iterative PSF smoothing (blue line, see text) or low band-pass pre-filtering of data (green line). The last three curves have been shifted for clarity. Beware that the left scale is logarithmic. (b) Log-log normalized power spectra of the upper panel curves. Power spectra are the modulus squared of the Fourier transform of the signal. The vertical dashed line pinpoints the frequency cutoff used in data filtering before LR deconvolution. The oscillations on graphs are due to the finite-size of the probed energy range.

(Fig. 4(a), green line). The used frequency cutoff (dashed line in Fig. 4(b)) is somewhat arbitrary. In Fig. 4(b), where plotted are the normalized power spectra of all the curves shown in Fig. 4(a), the cutoff demarcates the nearly constant Fourier components of noise at high frequency from the decaying ones of the signal at low frequency. In passing, by comparing the power spectra of the noisy data to the deconvoluted ones, the Fourier decomposition clearly demonstrates the enhancement of the high frequencies by more than one order of magnitude by the LR algorithm.

Complex regularization methods ${ }^{65}$ based on penalty function in the maximum likelihood have been developed to prevent noise amplification while still sharpening actual features. A simpler approach of noise stabilization which avoids the over-filtering of the signal is based on the iterative character of the LR process. ${ }^{35}$ At each step, the algorithm enhances the resolution and therefore the high frequency components of the power spectrum of the object. The idea is to adapt the filtering to the sharpness of the deconvoluted object; at the end of each LR blind loop $\left(k=1 \ldots N_{B}\right)$, the object $O^{k}(x)$ is replaced by its convolution with a Gaussian peak $G^{k}(x)$, i.e., $O_{G}^{k}$ $=G^{k}(x) \otimes O^{k}(x)$. The normalization to one of $G_{k}(x)$ insures the conservation of the intensity but its FWHM is chosen as a fraction of the elastic peak (1/6 in the chosen example) to avoid slowing down the restoration process and over smoothing. Although apparent in Fig. 4(a), the benefit of this adaptative approach of filtering shows up better in the Fourier space in Fig. 4(b). While the filtering of initial data (Fig. 4(b), green line) improves the agreement with the clean data (Fig. 4(b), orange line) compared to the noisy one (Fig. 4(b), red line), nearly one order of magnitude in reciprocal units is gained by the adaptive filtering method (Fig. 4(b), blue line) in the matching with the expected clean data. As shown in Fig. 5, this is however at the expense of a lower convergence rate for the enhancement of the features. The FWHM of $O[l]_{N_{O}}^{k}$ is nearly $1 \mathrm{meV}$ higher than with noisy or clean data and requires two extra outer $l$-loops $((6 \times 30 \times 1 \times 1)$ instead of $(4 \times 30 \times 1 \times 1))$ to reach an identical final result. This roots into the adaptive process which, aside smoothing, broadens the curves by convolution with a Gaussian $G^{k}(x)$. This appears as an overestimation of the FWHM of the PSF larger than the initial one ( $8.3 \mathrm{meV}$ instead of $8 \mathrm{meV}$; red line in Fig. 4(a)). Of course, on an overall point of view, noise worsens considerably the agreement between the reconvoluted object $P_{t}[l] \otimes O[l]_{N_{O}}^{k}$ and the initial data (symbols compared to lines in Fig. 5(a)). But adaptive filtering does not deteriorate too much the figures of merit compared to the noisy data (dotted versus full lines in Fig. 5(a)).

Although recursive equations similar to Eq. (4) and based on maximum likelihood can be derived for other types of noise statistics ${ }^{28}$ the effect of non-Poisson noise in LR process deserves discussion. In Fig. 6, LR deconvolution is applied to data corrupted with normal noise with standard deviation equal to the signal intensity which compares to Poisson noise (Fig. 6, red line). Overall, iterative noise stabilization procedure behaves as satisfactorily as in the case of Poisson noise (Fig. 6, blue line) in terms of enhancement of the main features, convergence speed, and final elastic peak FWHM. 


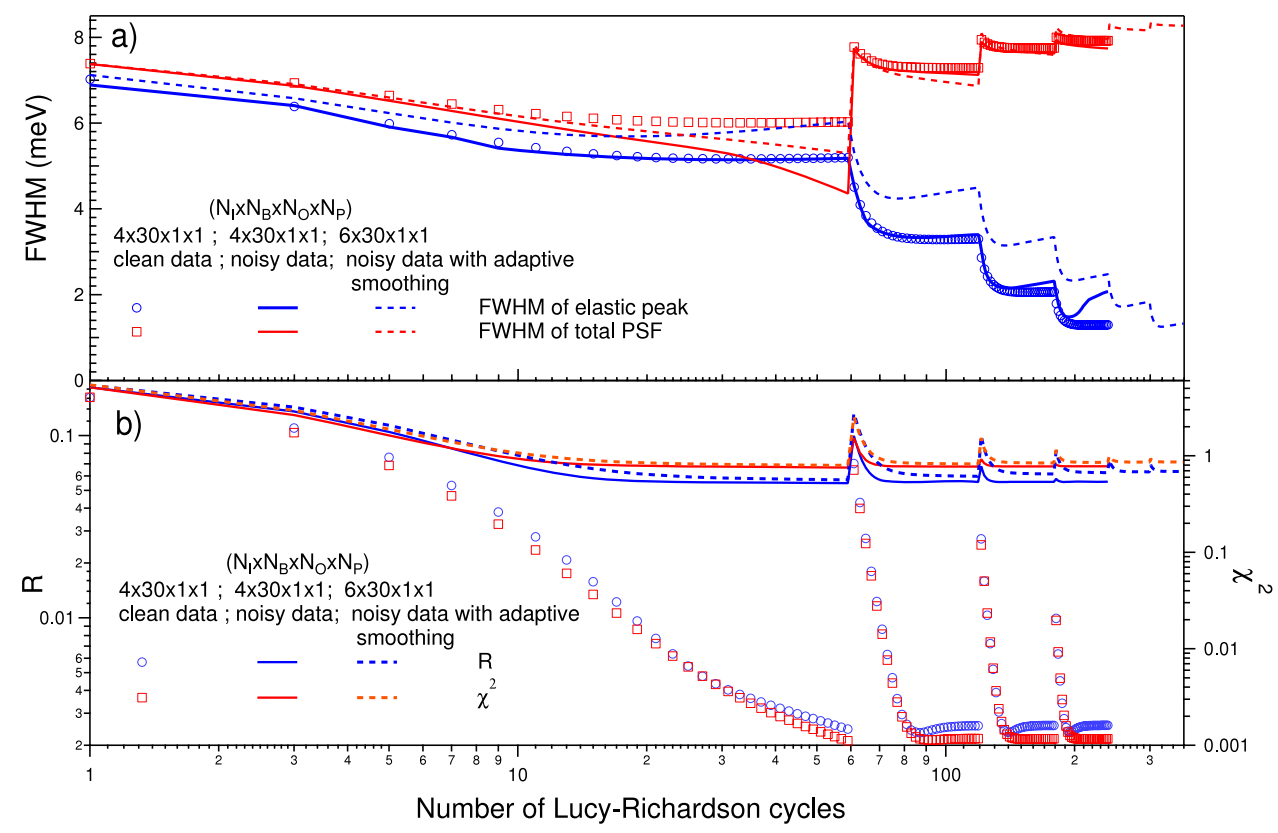

FIG. 5. Convergence rates of (a) FWHMs of elastic peak and of total PSF and (b) of the figures of merit $R$, $\chi^{2}$ (Eqs. (10)) during the semi-blind deconvolution of noisy spectra. The evolution for the clean data (symbols), the noisy data (full lines), and the adaptive filtering (dotted lines) are compared. The graphs are similar to those of Fig. 3 but applied to data of Fig. 4.

However, the agreement factor $\left(\chi^{2}(\right.$ Normal $) / \chi^{2}($ Poisson $\left.) \simeq 4\right)$ is worse. The filtering of speckles (diamond symbols; Fig. 6) is less efficient than with Poisson noise and leads to fake deconvoluted peaks. Therefore, although the deconvolution seems stable towards the type of noise statistics, caution should be exercised in feature assignment by playing with the degree of filtering.

\section{Point-spread function shape recovery}

The question which arises now is the ability of the algorithm not only to recover the signal but also the initial PSF. Despite its theoretical triangular shape in a cylindrical monochromator, ${ }^{1,4}$ the resolution function often becomes Gaussian because of averaging over angular spread of electron trajectories around the nominal central path, surface roughness, and monochromator/analyzer settings. However, angular aberrations in the optics contribute also to low energy tail and elastic peak asymmetry. ${ }^{1,4}$ Different initial shapes are compared in
Fig. 7, namely, a Gaussian $P_{G}(x)=1 / \sigma_{G} \sqrt{2 \pi} \exp \left[-x^{2} / 2 \sigma_{G}^{2}\right]$ $\left(\mathrm{FWHM}=2 \sqrt{2 \ln 2} \sigma_{G}\right)$, a Lorentzian $P_{L}=\sigma_{L} / \pi\left[\sigma_{L}^{2}+x^{2}\right]$ $\left(\mathrm{FWHM}=\sigma_{L}\right)$, and an asymmetric shape $P_{A}(x)=P_{G}(x)$ $\otimes P_{E}(x)$ which is calculated as the folding product of a Gaussian $P_{G}(x)$ and of an exponential decay $P_{E}(x>0)$ $=\exp \left(-x / \lambda_{E}\right) ; P_{E}(x<0)=0$. To allow comparison, the starting FWHM $(8 \mathrm{meV})$ of the elastic peak of the $\mathrm{TiO}_{2}$ test case is the same and the deconvolution $(+\infty \times 30 \times 1 \times 1)$ is stopped at the same final FWHM $(1.5 \mathrm{meV})$. At each semi-blind loop $l$, the trial PSF $P[l+1]_{0}^{0}(x)$ is reset as a Gaussian of FWHM larger or smaller than the elastic peak at the end of the cycle $l$. Fig. 7 demonstrates that the algorithm converges towards the initial PSF, irrespective of its profile. The final $P_{t}(x)$ closely resembles the initial $P(x)$ provided the final elastic peak is not a perfect Dirac $($ FWHM $=1.5 \mathrm{meV})$ and similar final deconvoluted objects are obtained (not shown). Moreover, the deconvolution is quite stable towards the shapes and widths of the starting function $P[l+1]_{0}^{0}(x)$ to the condition that its width encompasses the initial broadening. For a Gaussian

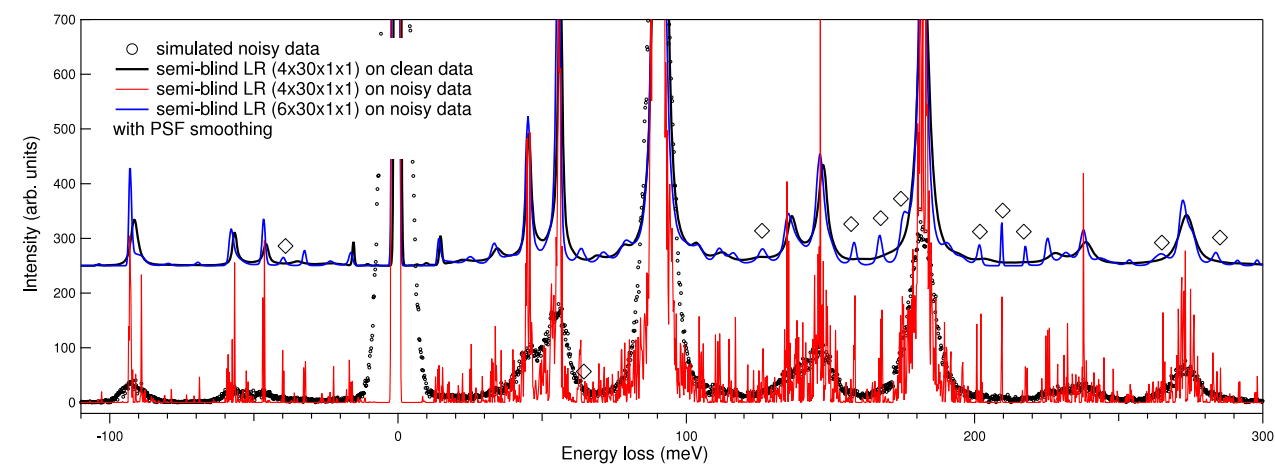

FIG. 6. Same figure as Fig. 4(a) but with data corrupted with normal noise instead of Poisson noise. Despite noise filtering, some speckles are amplified by the deconvolution process (diamonds symbols). 


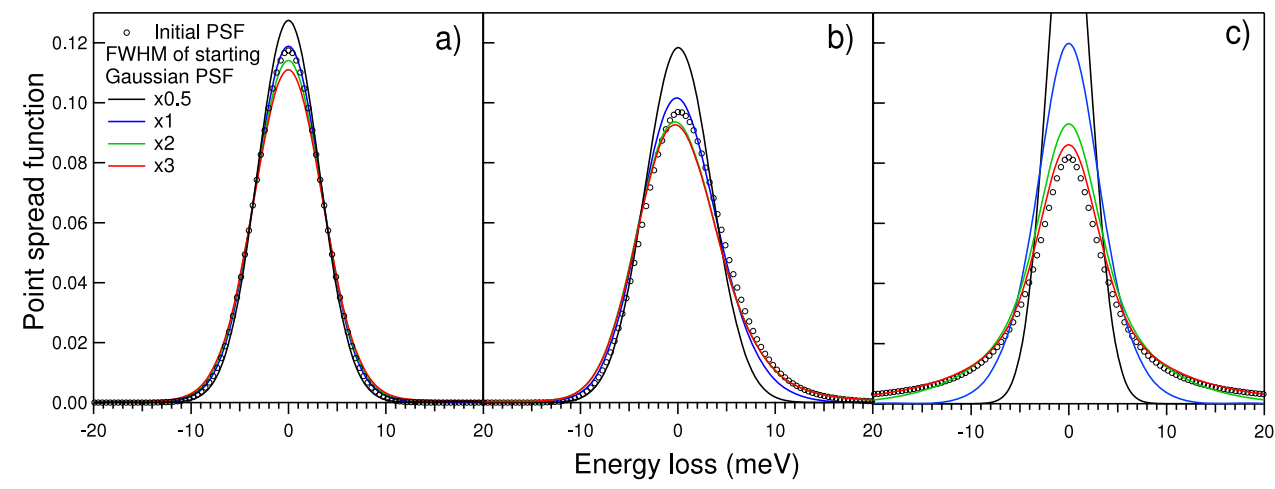

FIG. 7. PSF shape recovery after semi-blind LR deconvolution for three different initial PSF shapes (symbol, FWHM $=8$ meV): (a) Gaussian, (b) asymmetric Gaussian, and (c) Lorentzian. The trial PSF of the deconvolution at each semi-blind step $P[l+1]_{0}^{0}(x)$ is a Gaussian whose FWHM is either $0.5,1,2,3$ times that of the elastic peak. The $(\infty \times 30 \times 1 \times 1)$ process has been stopped at the same final FWHM $(1.5 \mathrm{meV})$ of the deconvoluted elastic peak.

PSF (Fig. 7(a)), convergence towards the solution is hardly sensitive to the width of the trial PSF $P[l+1]_{0}^{0}(x)$ while, in the extreme case of a Lorentzian shape (Fig. 7(c)), very broad trial solutions are required.

\section{E. Low-energy losses and deconvolution: The plasmon case}

The closeness of energy losses from the elastic peak may prevent their correct deconvolution. ${ }^{11} \mathrm{~A}$ case of relevance is the surface plasmon excitation of the conduction electrons. Its energy $\omega_{P, s}=\omega_{P} / \sqrt{1+\epsilon(0)}$ is related to the bulk plasmon frequency $\omega_{P}$ and the static dielectric constant $\epsilon(0)$. Typical carrier concentrations in titania lead to $\omega_{P, s}$ values ranging between a fraction of $\mathrm{meV}$ and several tens of $\mathrm{meV}$. As shown theoretically and experimentally in semi-conductors, ${ }^{1,42}$ if $\omega_{P, s}$ is smaller than the experimental resolution, plasmon multiple excitations give rise to a continuous broadening of the elastic peak. For higher carrier concentration, the plasmon excitation can appear as a distinct feature, screening and mixing with phonons leading to quasi-particles known as "plasmaron." ${ }^{66-68}$ The underlying question is the capability of the algorithm to recover those features and to distinguish them from the PSF. In Fig. 8, the semi-blind LR algorithm has been applied to simulations including both phonon and plasmon contributions accordingly to the dielectric function given by Eq. (14). The used values $\omega_{P}=100 \mathrm{meV}$ and $\gamma_{p}=10 \mathrm{meV}$ are typical of carrier concentration due to vacancies and interstitial doping in $\mathrm{TiO}_{2}$. Compared to undoped $\mathrm{TiO}_{2}$ (Fig. 1), carriers give rise to shift and broadening of phonon peaks. In particular, the elastic peak has a typical asymmetric shape due to the Boltzmann occupancy factor. Care should be taken in the choice of the initial PSF $P[l]_{0}^{0}$ of the semi-blind loop. With a Gaussian peak of FWHM, one-half smaller than the width of the $O[l-1]_{N_{O}}^{N_{B}}$ elastic peak, data are perfectly recovered as shown by the overlap with a simulation using a better resolution (red line versus blue dotted line in Fig. 8). However, other starting PSF such as a broader Gaussian peak or the choppedout initial elastic peak gives different final results with sharper phonon peaks. With the first solution (green line of Fig. 8),

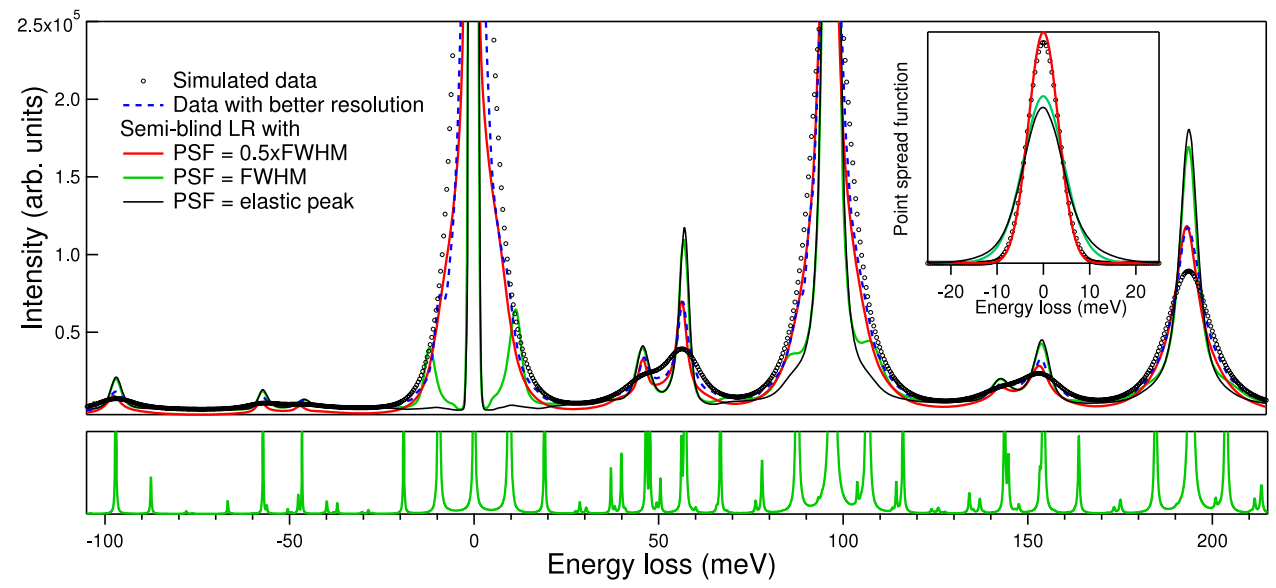

FIG. 8. Simulated phonon spectrum of $\mathrm{TiO}_{2}$ with low-energy plasmon losses $\left(\omega_{p}=100 \mathrm{meV}, \Gamma_{p}=10 \mathrm{meV}\right)$ (circles) and its semi-blind deconvolution within different trial PSF $P[l]_{0}^{0}$ : (a) a Gaussian peak of the same width than that of the elastic peak $O[l-1]_{N_{O}}^{N_{B}}$ (green line) or (b) smaller (1/2)(red line) and (c) the elastic peak chopped out from the spectrum (black line). The $(\infty \times 30 \times 1 \times 1)$ process has been stopped at the same final FWHM $(1.5 \mathrm{meV})$ of the deconvoluted elastic peak. Deconvoluted spectra are compared with a simulation with a better resolution ( $1.5 \mathrm{meV}$ instead of $8 \mathrm{meV}$; blue dotted line). The position of the expected phonon/plasmon lines is displayed in the lower panel. The initial Gaussian PSF (circles) and the obtained ones after deconvolution with the same labeling are shown in the inset. 
wings pop-up at the foot of the elastic peak or of the intense phonon features. Their position does not match perfectly the expected frequencies of the surface plasmon (lower panel of Fig. 8) but their intensity ratio matches the Boltzmann factor.
This discrepancy with Sec. IV D about the PSF shape recovery is only apparent as it lies in the indeterminacy of what is signal and what is PSF. Factorizing Eq. (15) with phonon $S_{p h}(\hbar \omega)$ and plasmon $S_{p l}(\hbar \omega)$ single excitation

$$
\begin{aligned}
I(\hbar \omega) & \left.=P(\hbar \omega) \otimes\left\{\delta(\hbar \omega)+S_{p l}(\hbar \omega)+S_{p h}(\hbar \omega)+\frac{1}{2 !}\left[S_{p l}(\hbar \omega)+S_{p h}(\hbar \omega)\right] \otimes\left[S_{p l} \hbar \omega\right)+S_{p h}(\hbar \omega)\right]+\cdots\right\} \\
& =P(\hbar \omega) \otimes\left\{\delta(\hbar \omega)+S_{p l}(\hbar \omega)+\frac{1}{2 !} S_{p l}(\hbar \omega) \otimes S_{p l}(\hbar \omega)+\cdots\right\} \otimes\left\{\delta(\hbar \omega)+S_{p h}(\hbar \omega)+\frac{1}{2 !} S_{p h}(\hbar \omega) \otimes S_{p h}(\hbar \omega)+\cdots\right\} \\
& =P(\hbar \omega) \otimes S_{m, p l}(\hbar \omega) \otimes S_{m, p h}(\hbar \omega),
\end{aligned}
$$

shows that, in the framework of the LR algorithm, $P(\hbar \omega)$ $\otimes S_{m, p l}(\hbar \omega)$ can be seen as the PSF of $S_{m, p h}(\hbar \omega)$. Therefore, a priori knowledge about the initial PSF drives the solution towards a defined solution. If the initial PSF $P[l]_{0}^{0}$ is close to the elastic peak shape, that is to say $P(\hbar \omega) \otimes\{\delta(\hbar \omega)$ $\left.+S_{p l}(\hbar \omega)\right\} \simeq S_{m, p l}(\hbar \omega)$, a solution close to $S_{m, p h}(\hbar \omega)$ (black line), without any plasmon losses but still with phonon screening and shifts, is recovered. For a sharper $P[l]_{0}^{0}$ peak, the algorithm converges towards the sought solution $S_{m, p l}(\hbar \omega) \otimes$ $S_{m, p h}(\hbar \omega)$ and the right PSF (blue versus red lines in Fig. 8). For a $P[l]_{0}^{0}$ Gaussian peak of the same FWHM as the elastic peak of $O[l-1]_{N_{O}}^{N_{B}}$ (green line in Fig. 8), the final result is intermediate but the asymmetry due to temperature occupancy factor points to the existence of a plasmon feature.

In Fig. 9, the same procedure of deconvolution is applied to a simulated phonon spectrum with an asymmetric PSF $P_{A}(x)=P_{G}(x) \otimes \exp \left(-x / \lambda_{E}\right)\left(\lambda_{E}=5 \mathrm{meV}\right)$ but without plasmon contribution, i.e., $I(\hbar \omega)=P_{A}(x) \otimes S_{m, p h}(\hbar \omega)$. The asymmetry of the resolution function of HREELS ${ }^{1,4}$ is a common artefact due to the complex electron trajectories in monochromator and analyzer. The same questioning appears: should the asymmetric exponential decay be included in the object or the PSF? Besides that intrinsic indeterminacy, the LR deconvolution helps deciphering between various contributions in elastic peak shape by enhancing the apparent resolution. The lack of the gain peak and the asymmetry of all phonon peaks (Fig. 9) rules out any plasmon contribution.

\section{APPLICATION OF THE SEMI-BLIND DECONVOLUTION TO EXPERIMENTAL TiO ${ }_{2}(110)$ HREELS SPECTRA}

The semi-blind LR approach has been applied to an experimental HREELS spectrum of $\mathrm{TiO}_{2}(110)$ (Figs. 10 and 11) acquired in the specular geometry. The counting rate at elastic peak maximum is $528 \mathrm{kHz}$ with a FWHM of $12 \mathrm{meV}$ and a step size $\Delta \hbar \omega=0.305 \mathrm{meV}$. The $(\infty \times 30 \times 1 \times 1)$ deconvolution was carried out until reaching an apparent elastic FWHM of $2 \mathrm{meV}$ for $O[l]_{j}^{k}$. Following results of Sec. IV E, three different starting PSF $P[l]_{0}^{0}$ are compared: (i) Gaussian peaks with a FWHM equal 1 (green curve, Figs. 10 and 11) or 1/2 (red curve, Figs. 10 and 11) times that of $O[l-1]_{N_{P}}^{N_{B}}$ or (ii) the elastic peak itself chopped-out from the spectrum (black curve, Figs. 10 and 11(a)). Regardless of the starting point, a sizeable enhancement of the resolution is obtained. As checked carefully in Sec. IV E, the choice of a trial PSF narrower than the elastic peak shape (red curve, Figs. 10 and 11(a)) allow a clear restoration of peaks and shoulders (stars, Figs. 10 and 11(a)) due to multiple excitations and combinations of phonon and plasmon-like surface excitation. This assignment

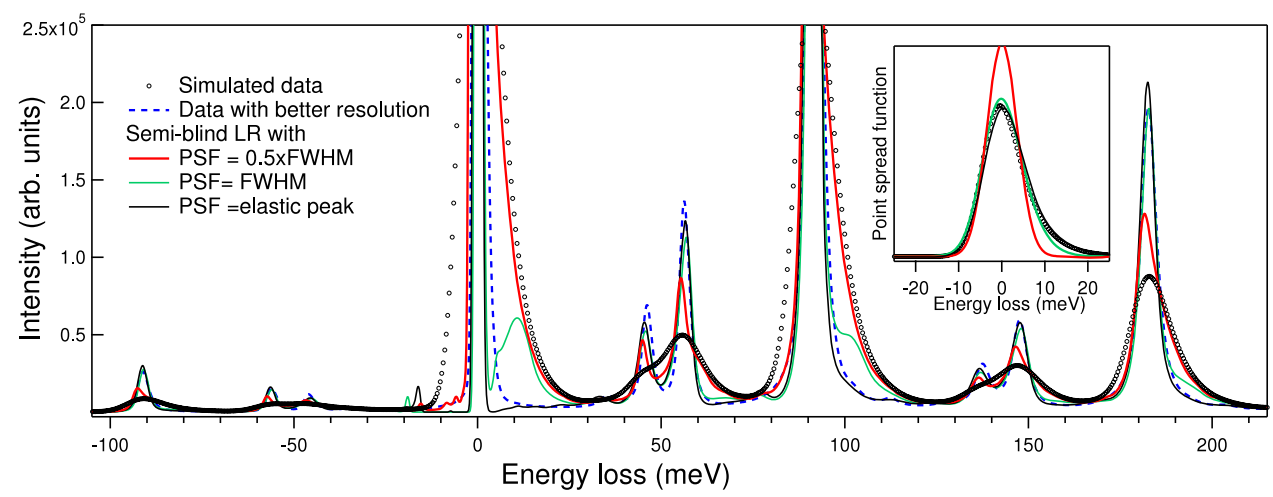

FIG. 9. Same as Fig. 8 without plasmon contribution but with an asymmetric initial PSF $P_{A}(x)=P_{G}(x) \otimes P_{E}(x)$ defined as the folding product of a Gaussian $P_{G}(x)$ and an exponential decay $P_{E}(x>0)=\exp \left(-x / \lambda_{E}\right) ; P_{E}(x<0)=0(\lambda=5$ meV). The simulation with a better resolution is obtained by reducing both Gaussian FWHM of $P_{G}$ and $\lambda_{E}$. 


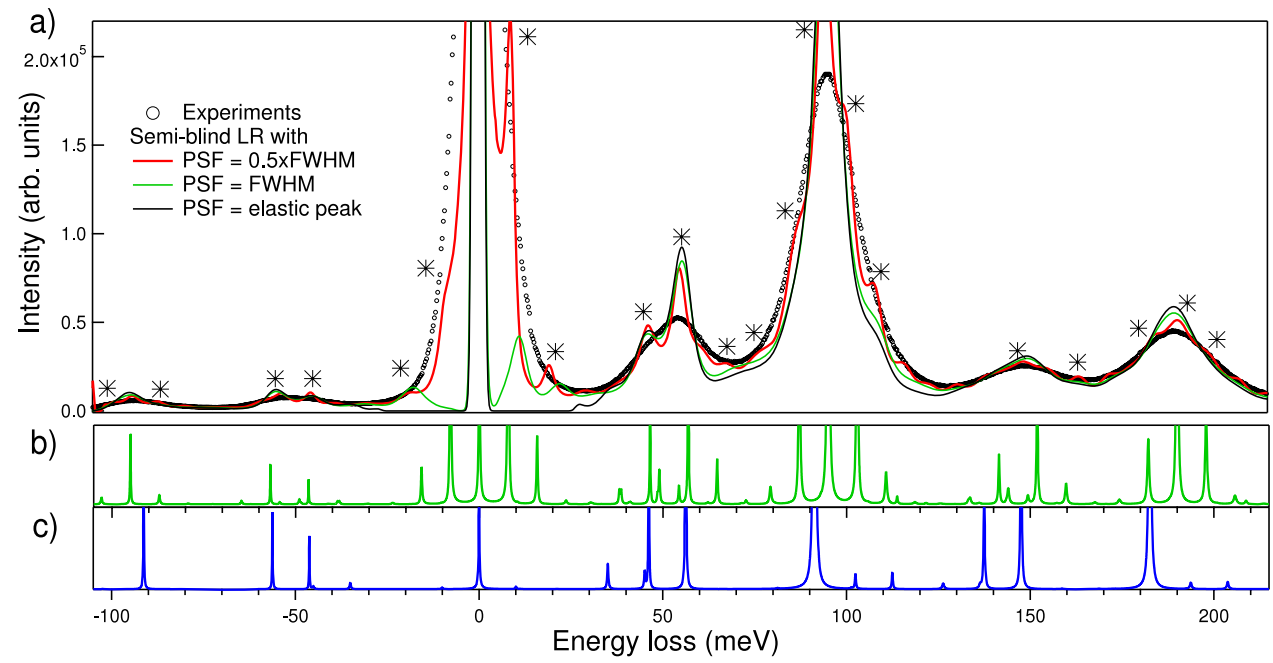

FIG. 10. (a) Semi-blind LR deconvolution of a HREELS spectrum of $\mathrm{TiO}_{2}(110)$ (circles) with different trial initial PSF $P[l]_{0}^{0}$ : (i) Gaussian with a FWHM one-half (red line) smaller or (ii) equal (green line) to that $O[l-1]_{N_{O}}^{N_{B}}$ or (iii) the chopped-out experimental elastic peak (black line). The $(\infty \times 30 \times 1 \times 1) \mathrm{LR}$ process has been stopped at the same elastic peak FWHM of $O[l]_{k}^{j}(2 \mathrm{meV})$ and stabilized against noise amplification accordingly to Sec. IV C (see Fig. 12). (b) Simulated $\mathrm{TiO}_{2}$ HREELS spectrum with undamped phonon and plasmon $\left(\omega_{P}=80 \mathrm{meV}\right)$ contributions and no instrumental broadening. (c) Same but without plasmon $\omega_{P}=0$. The main features revealed by the resolution enhancement process are pinpointed by stars.

is clearly confirmed by comparison with model spectra for which the plasmon $\gamma_{P}$ and phonon $\gamma_{n}$ parameters as well as the experimental broadening are purposely reduced by two orders of magnitude (Figs. 10-11(c)). With a bulk plasma value of $\omega_{P}=80 \mathrm{meV}$, the expected number of peaks and their respective positions matches the deconvolution findings. The one to one correspondence with the simulation assesses the relevance of the deconvolution. The part of the spectrum corresponding to the first harmonics of the phonon spectrum is quite successfully achieved, with a doublet at $\sim \pm 50 \mathrm{meV}$ and a multifeatured peak at $95 \mathrm{meV}$. Discrepancies with simulated spectra are observed at higher energy (in particular, above $220 \mathrm{meV}$ where multiple excitations and combination bands dominate the spectra) may be due to the hypothesis of flat profile of carrier concentration (Eq. (14)). The presence of plasmon contributions is further supported by (i) the shifts of the absolute positions of the phonon peaks by several $\mathrm{meV}$ compared to $\omega_{P}=0$ case (Figs. 10-11(b) vs 11 (c)) due to the screening by plasmons and (ii) the initial strong asymmetry of the experimental elastic peak. Similar conclusion and $\omega_{P}$ values were found by infra-red reflectivity analysis ${ }^{45,46,64}$ and transport measurements. ${ }^{61-63}$ Other choices of initial $P[l]_{0}^{0}$ are possible. As explained in Sec. IV E, those choices may lead to either a washout of the plasmon contribution (black curve, Figs. 10 and 11(a)) which results in only shifted phonon peaks or to an intermediate situation (green curve, Figs. 10 and 11(a)) for which the algorithm has transferred plasmonic signal towards the experimental PSF. As demonstrated in Fig. 12(b), noise filtering is mandatory to avoid amplification during the deconvolution process. As suggested in Sec. IV C, the signal is convoluted at each semi-bling loop $l$ by a normalized Gaussian of width equal to a fraction of the elastic peak of $O[l-1]_{N_{O}}^{N_{B}}$ ( $1 / 6$ in the present case). Lack of filtering is catastrophic (black line, Fig. 12(b)) but a compromise should be found between

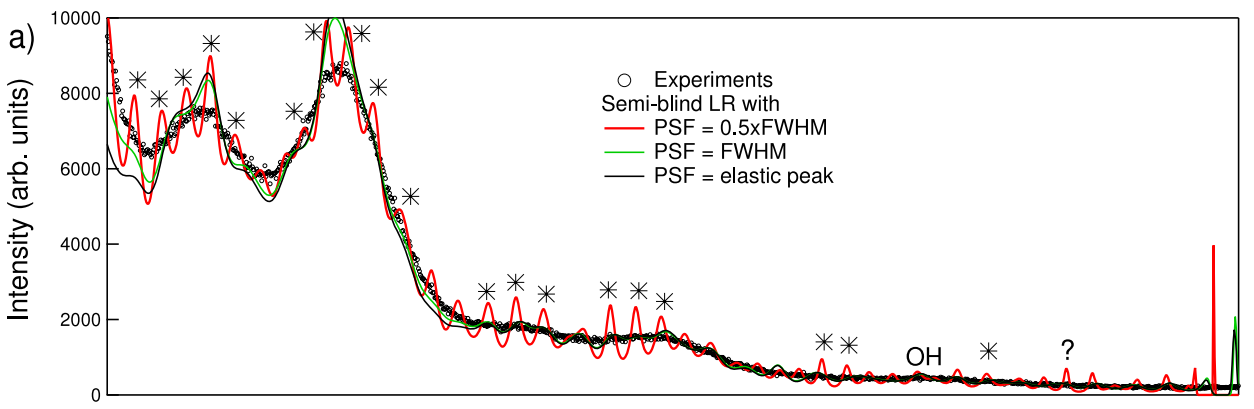

b)

c)

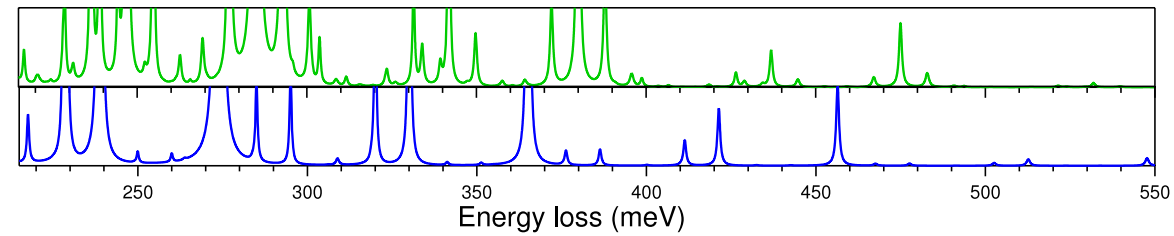

FIG. 11. Same as Fig. 10 but on the $220-550 \mathrm{meV}$ energy loss scale. Besides a shift, the enhanced peaks marked by stars match qualitatively with the predicted ones in the framework of dielectric modeling (see text). In particular, notice the two excitation triplets between 320-380 meV revealed by deconvolution. 

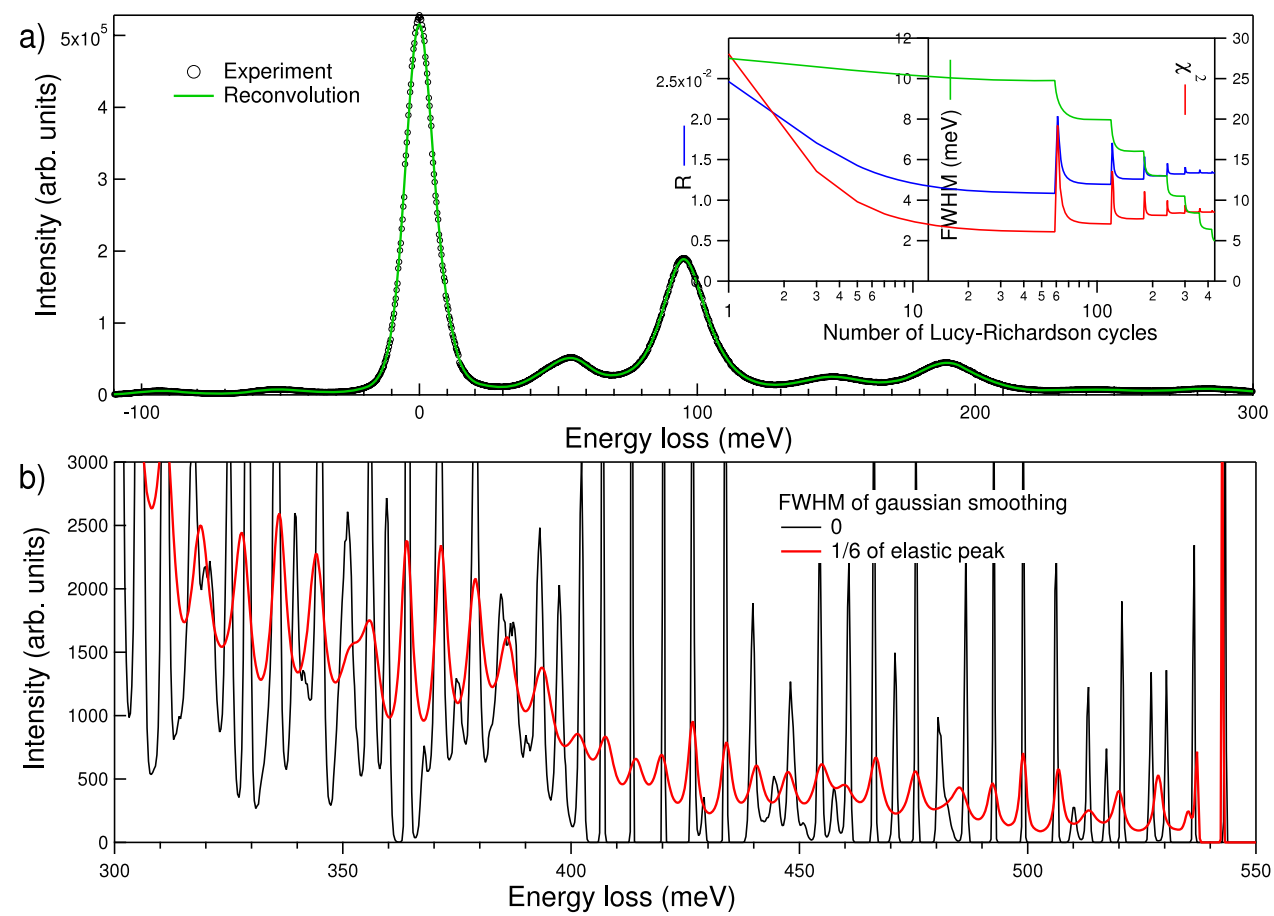

FIG. 12. (a) Comparison between the experimental $\mathrm{TiO}_{2}$ HREELS spectrum (circles) and the convolution product of total final PSF $P_{t}(x)$ and the deconvoluted object $O\left[N_{I}\right]_{N_{P}}^{N_{B}}$ (red curve of Figs. 10 and 11). The convergence with the total number of LR cycles of the agreement factor $R$ (left scale, blue curve), $\chi^{2}$ (right scale, red curve) and the elastic peak $O[l]_{k}^{j}$ FWHM is shown in inset. (b) Semi-blind LR deconvoluted curves without (black line) and with (red line) noise filtering with the Gaussian convolution technique of Sec. IV C. The FWHM of the folding Gaussian is $1 / 6$ of the FWHM of the elastic peak of $O[l]_{N_{P}}^{N_{B}}$

signal enhancement and noise damping. With broader smoothing Gaussian (not shown), the restoration process is slowed down and the visibility of the plasmon features is reduced. The reconvoluted spectrum $P_{t}(x) \otimes O\left[N_{I}\right]_{N_{O}}^{N_{B}}$ (Fig. 12(a)) matches nearly perfectly the experimental with agreement factors of $R=0.015, \chi^{2}=8.7$ at the end of deconvolution (Fig. 12(a), inset).

\section{CONCLUSION}

A new algorithm based on iterative LR approach was proposed for the spectral restoration of HREELS data. Provided that the resolution is constant over all the spectra, the method extracts, in a semi-blind way, the PSF and the resolution enhanced data. Since the elastic peak does not provide a reliable template of the apparatus function, the algorithm offers the opportunity to recover low energy excitations hidden in the so called "quasi-elastic." This was illustrated in the case of mixed multiple excitation in $\mathrm{TiO}_{2}$ both on synthetic and experimental spectra. The algorithm helps efficiently improving the resolution in HREELS measurements.

\section{ACKNOWLEDGMENTS}

L. De Caro (CNR, Italy) is acknowledged for fruitful discussion about deconvolution methods.

${ }^{1}$ H. Ibach and D. L. Mills, Electron Energy Loss Spectroscopy and Surface Vibrations (Academic Press, New York, 1982).

${ }^{2}$ P. A. Thiry, M. Liehr, J.-J. Pireaux, and R. Caudano, Phys. Scr. 35, 368 (1987).
${ }^{3}$ Y. J. Chabal, Surf. Sci. Rep. 8, 211 (1988).

${ }^{4} \mathrm{H}$. Ibach, Electron Energy Loss Spectrometers: The Technology of High Performance (Springer-Verlag, 1991).

${ }^{5}$ B. G. Frederick, G. L. Nyberg, and N. V. Richardson, J. Electron Spectrosc. Relat. Phenom. 64-65, 825 (1993).

${ }^{6} \mathrm{H}$. Ibach, M. Balden, D. Bruschmann, and S. Lehwal, Surf. Sci. 269-270, 94 (1992).

${ }^{7}$ See www.specs.de for Delta 0.5 HREELS spectrometer.

${ }^{8}$ P. A. Cox, W. R. Flavell, A. A. Williams, and R. G. Egdell, Surf. Sci. 152153, 784 (1985).

${ }^{9}$ W. T. Petrie and J. M. Vohs, Surf. Sci. 245, 315 (1991).

${ }^{10}$ M. A. Henderson, Surf. Sci. 355, 151 (1996).

${ }^{11}$ B. G. Frederick, B. B. Frederick, and N. V. Richardson, Surf. Sci. 368, 82 (1996).

${ }^{12}$ G. Apai and W. P. McKenna, Langmuir 7, 2266 (1991).

${ }^{13}$ B. G. Frederick, M. R. Ashton, and N. V. Richardson, Surf. Sci. 292, 33 (1993b).

${ }^{14}$ B. G. Frederick, G. Apai, and T. N. Rhodin, Phys. Rev. B 44, 1880 (1991).

${ }^{15}$ Y. Wang and W. H. Weinberg, Phys. Rev. Lett. 69, 3326 (1992a).

${ }^{16}$ Y. Wang and W. H. Weinberg, Surf. Sci. 287-288, 1102 (1992b).

${ }^{17}$ R. Narayan and R. Nityananda, Annu. Rev. Astron. Astrophys. 24, 127 (1986).

${ }^{18}$ G. Tisdale and S. J. Sibener, Surf. Sci. 311, 360 (1994).

${ }^{19}$ W. von der Linden, V. Dose, R. Matzdor, A. Pantförder, G. Meister, and A. Goldmann, J. Electron Spectrosc. Relat. Phenom. 83, 1 (1997).

${ }^{20}$ A. A. Aquino, C. P. A. Mulcahy, and T. S. Jone, Surf. Sci. 344, L1231 (1995).

${ }^{21}$ K. Jacobi, M. Gruyters, P. Geng, T. Bitzer, M. Aggour, S. Rauscher, and H. J. Lewerenz, Phys. Rev. B 51, 5437 (1995).

${ }^{22}$ K. Jacobi, Y. Wang, C. Y. Fan, and H. Dietrich, J. Chem. Phys. 115, 4306 (2001).

${ }^{23}$ L. L. Kesmodel, S. Wild, and G. Apai, Surf. Sci. 429, L475 (1999).

${ }^{24}$ M. Eremtchenko, S. Döring, R. Temirov, and J. A. Schaefer, Phys. Rev. B 71, 045410 (2005).

${ }^{25}$ R. T. Seljamäe-Green, G. J. Simpson, F. Grillo, J. Greenwood, S. M. Francis, R. Schaub, P. Lacovig, and C. J. Baddeley, Langmuir 30, 3495 (2014).

${ }^{26}$ C. Charles, G. Leclerc, J.-P. Rasson, and J.-J. Pireaux, Surf. Interface Anal. 36, 61 (2004). 
${ }^{27}$ G. M. P. Van Kempen, L. J. van Vliet, P. J. Verveer, and H. T. M. Van der Voort, J. Microsc. 185, 354 (1997).

${ }^{28}$ J. L. Starck and E. Pantin, Publ. Astron. Soc. Pac. 114, 1051-1069 (2002).

${ }^{29}$ W. Richardson, J. Opt. Soc. Am. 62, 55 (1972).

${ }^{30}$ L. Lucy, Astron. J. 79, 745 (1974).

${ }^{31}$ N. Dey, L. Blanc-Feraud, C. Zimmer, P. Roux, Z. Kam, and J. C. OlivoMarin, Microsc. Res. Tech. 69, 260 (2006).

${ }^{32}$ A. Gloter, A. Douiri, M. Tencé, and C. Colliex, Ultramicroscopy 96, 385 (2003).

${ }^{33}$ R. F. Egerton, H. Qian, and M. Malac, Micron 37, 310 (2006).

${ }^{34}$ W. E. McBride, D. J. H. Cockayne, and C. M. Goringe, Ultramiscroscopy 76, 115 (1999).

${ }^{35}$ T. T. Fister, G. T. Seidler, J. J. Rehr, J. J. Kas, W. T. Elam, J. O. Cross, and K. P. Nagle, Phys. Rev. B 75, 174106 (2007).

${ }^{36}$ J. D. Rameau, H.-B. Yang, and P. D. Johnson, J. Electron Spectrosc. Relat. Phenom. 181, 35 (2010).

${ }^{37}$ L. De Caro, F. A. Altamura, D. Vittoria, G. Carbone, F. Qiao, L. Mannac, and C. Giannini, J. Appl. Cryst. 45, 1228 (2012).

${ }^{38}$ L. Kesmodel, J. Vac. Sci. Technol., A 1, 1456 (1983).

${ }^{39}$ F. Tsumuraya, J. Opt. Soc. Am. A 13, 1532 (1996).

${ }^{40}$ R. G. Lan, J. Opt. Soc. Am. A 13, 1992 (1996).

${ }^{41}$ F. Tsumuraya, N. Miura, and N. Baba, Astron. Astrophys. 282, 699 (1994); available at http://adsabs.harvard.edu/abs/1994A\%26A...282..699T.

${ }^{42}$ B. N. J. Persson and J. E. Demuth, Phys. Rev. B 30, 5968 (1984).

${ }^{43}$ B. N. J. Persson and E. Zaremba, Phys. Rev. B 31, 1863 (1985).

${ }^{44}$ D. A. Fish, A. M. Brinicombe, E. R. Pike, and J. G. Walker, J. Opt. Soc. Am. A 12, 58 (1995).

${ }^{45}$ J. F. Baumard and F. Gervais, Phys. Rev. B 15, 2316 (1977).

${ }^{46}$ E. Hendry, F. Wang, J. Shan, T. F. Heinz, and M. Bonn, Phys. Rev. B 69, 081101(R) (2004).
${ }^{47}$ A. A. Lucas and M. Sunjić, Prog. Surf. Sci. 2, 75 (1972).

${ }^{48}$ D. Mills, Surf. Sci. 48, 59 (1975).

${ }^{49}$ S. Eriksen and R. G. Egdell, Surf. Sci. 180, 263 (1987).

${ }^{50}$ U. Diebold, Surf. Sci. Rep. 48, 53 (2003).

${ }^{51}$ E. D. Palik, Handbook of Optical Constants of Solids (Academic Press, 1985), Vol. 1-3.

${ }^{52}$ A. A. Lucas and J. P. Vigneron, Solid State Commun. 49, 327 (1984).

${ }^{53}$ D. M. Eagles, J. Phys. Chem. Solids 25, 1243 (1964).

${ }^{54}$ J. G. Traylor, H. G. Smith, R. M. Nicklow, and M. K. Wilkinson, Phys. Rev. B 3, 3457 (1971).

${ }^{55}$ C. Lee, P. Ghosez, and X. Gonze, Phys. Rev. B 50, 13379 (1994).

${ }^{56}$ H. Ibach, Phys. Rev. Lett. 24, 1416 (1970).

${ }^{57}$ G. Mattioli, P. Alippi, F. Filippone, R. Caminiti, and A. A. Bonapasta, J. Phys. Chem. C 114, 21694 (2010).

${ }^{58}$ A. Janotti, C. Franchini, J. B. Varley, G. Kresse, and C. G. Van de Valle, Phys. Status Solidi RRL 7, 199 (2013).

${ }^{59}$ A. Fujimori, A. E. Bocquet, K. Morikawa, K. Kobayashi, I. T. Saithoh, Y. Tokura, I. Hasex, and M. Onoda, J. Phys. Chem. Solids 57, 1379 (1996).

${ }^{60}$ F. Gervais and J. F. Baumard, Solid State Commun. 21, 861 (1977).

${ }^{61}$ E. Iguchi, K. Yajima, T. Asahina, and Y. Kanamori, J. Phys. Chem. Solids 35, 597 (1974).

${ }^{62}$ R. R. Hasiguti and E. Yagi, Phys. Rev. B 49, 7251 (1994).

${ }^{63}$ E. Yagi, R. R. Hasiguti, and M. Aono, Phys. Rev. B 54, 7945 (1996).

${ }^{64}$ D. A. Panayotov, S. P. Burrows, and J. R. Morris, J. Phys. Chem. C 116, 4535 (2012)

${ }^{65}$ T. F. Chan and C. K. Wong, IEEE Trans. Image Process. 7, 370 (1998).

${ }^{66}$ J. I. Gersten, Surf. Sci. 92, 579 (1980).

${ }^{67}$ Y. Goldstein, A. Many, and I. Wagner, Surf. Sci. 98, 599 (1980).

${ }^{68}$ A. Many, I. Wagner, A. Rosenthal, J. I. Gersten, and Y. Goldstein, Phys. Rev. Lett. 46, 1648 (1981). 